Pacific Journal of Mathematic 


\section{LIMITS IN CERTAIN CLASSES OF ABSTRACT ALGEBRAS}

\section{GloRIA C. HewitT}

This paper is primarily concerned with the existence of direct limits in certain classes of Boolean algebras. The concepts of inverse and direct limits are defined relative to a class $\mathfrak{A}$ of abstract algebras. It is assumed that the algebras in $\mathfrak{A}$ are of the same type.

It is found that classes which are closed under such constructions as the formation of homomorphic images, subalgebras, free products and free unions do admit direct and inverse limits. In fact, the existence of direct limits is closely related to the existence of free products and dually, the existence of inverse limits is related to the existence of free unions. Also there is a relationship between the existence of inverse limits and direct limits.

The proofs of these observations are omitted because they are similar in construction to the proofs given for existence theorems in $[2,3,5]$.

Let $\mathfrak{A}$ be a class of abstract algebras. Let $A$ be a directed set and let $X=\left\{X_{\alpha} \mid \alpha \in A\right\}$ be a subset of $\mathfrak{A}$. A system $\langle X ; \Pi\rangle$ consisting of the family $X$ and a family $\Pi$ of homomorphisms

$$
\pi_{\alpha}^{\beta}: X_{\alpha} \rightarrow X_{\beta} \text { for each } \alpha<\beta \text { in } A,
$$

is called a direct system over $A$ if $\pi_{\alpha}^{\alpha}$ is the identity map of $X_{\alpha}$ and $\pi_{\alpha}^{\delta}=\pi_{\beta}^{\delta} \pi_{\alpha}^{\beta}$ whenever $\alpha<\beta<\delta$. A system $\left\langle X_{\infty} ; \pi_{\alpha}\right\rangle_{\alpha} \in_{A}$ consisting of an algebra $X_{\infty}$ in $\mathfrak{A}$ and a family of homomorphisms $\pi_{\alpha}: X_{\alpha} \rightarrow X_{\infty}$ such that if $\alpha<\beta, \pi_{\beta} \pi_{\alpha}^{\beta}=\pi_{\alpha}$ is called the direct limit of $\langle X$; $\Pi\rangle$ if the following extension property is satisfied:

(E) if $g_{\alpha}: X_{\alpha} \rightarrow Z$ is a family of homomorphisms such that if $\alpha<\beta$ in $A, g_{\beta} \pi_{\alpha}^{\beta}=g_{\alpha}$, where $Z \in \mathfrak{A}$, then there is a unique homomorphism $g: X_{\infty} \rightarrow Z$ such that $g_{\alpha}=g \pi_{\alpha}$ for all $\alpha$ in $A$.

The concepts of inverse system over $A$ and inverse limit are dual to that of direct system over $A$ and direct limit respectively.

\section{Closure properties.}

THEOREM 1.1. If $\mathfrak{A}$ is closed under free unions and the formation of subalgebras, then $\mathfrak{A}$ is closed under inverse limits. 
As a converse to this theorem we have the following result.

TheOREM 1.2. Let $\left\{X_{\alpha} \mid \alpha \in A\right\}$ be a subset of $\mathfrak{A}$. Suppose

(1) $\mathfrak{U}$ is closed under inverse limits;

(2) Any finite subset of $\left\{X_{\alpha} \mid \alpha \in A\right\}$ admits a free union. Assume that for each $\beta \in A$, there exist an algebra $\bar{X}_{\beta} \in \mathfrak{A}$ and $a$ family of homomorphisms $h_{\alpha \beta}: \bar{X}_{\beta} \rightarrow X_{\alpha}$ for all $\alpha \in A$ with $h_{\alpha \alpha}$ onto. Then $\left\{X_{\alpha} \mid \alpha \in A\right\}$ admits a free union.

THEOREM 1.3. If $\mathfrak{A}$ is closed under free products and the formation of homomorphic images, then $\mathfrak{X}$ is closed under direct limits.

Conversely,

Theorem 1.4. Let $\left\{X_{\alpha} \mid \alpha \in A\right\}$ be a subset of $\mathfrak{A}$. Suppose

(1) $\mathfrak{U}$ is closed under direct limits;

(2) Any finite subset of $\left\{X_{\alpha} \mid \alpha \in A\right\}$ admits a free product. Assume that for each $\beta \in A$, there exist an algebra $\bar{X}_{\beta} \in \mathfrak{A}$ and $a$ family of homomorphisms $h_{\alpha \beta}: X_{\alpha} \rightarrow \bar{X}_{\beta}$ for all $\alpha \in A$ with $h_{\alpha \alpha}$ oneto-one. Then $\left\{X_{\alpha} \mid \alpha \in A\right\}$ admits a free product.

THeOREM 1.5. Suppose $\mathfrak{A}$ is closed under the formation of homomorphic images, free products of finite subsets, and direct limits. Then $\mathfrak{A}$ is closed under inverse limits.

Conversely,

THEOREM 1.6. Suppose $\mathfrak{A}$ is closed under isomorphism, the formation of subalgebras, inverse limits and free unions of finite subsets. Then $\mathfrak{U}$ is closed under direct limits.

We observe here that if $\mathfrak{A}$ is a class of abstract algebras which is closed under the formation of homomorphic images, subalgebras, direct unions and if every algebra of $\mathfrak{A}$ contains a one element subalgebra, then every inverse (direct) system in $\mathfrak{A}$ admits an inverse (a direct) limit. It is easy to see that if the direct union is in $\mathfrak{A}$, then it is the free union. This fact, together with 1.1, gives that every inverse system in $\mathfrak{U}$ admits an inverse limit. Sikorski (in [7]) proved that every subset of $\mathfrak{U}$ has a free product, (see also [2], p. 88). This, together with 1.3 , yields that every direct system in $\mathscr{U}$ admits a direct limit.

2. Direct limits in particular classes. For the results in this section, we will need the following fact. 
Lemma 2.1. Let $\left\langle X_{\infty} ; \pi_{\alpha}\right\rangle_{a} \in_{A}$ be a system having all the properties of the definition of a direct limit except possibly the uniqueness of the homomorphism $g$ in $(E)$. Assume that $\bigcup\left\{\pi_{a}\left[X_{\alpha}\right] \mid \alpha \in A\right\}$ generates $X_{\infty}$. Then $g$ is unique.

Conversely, if the class $\mathfrak{A}$ is closed under the formation of subalgebras and $\left\langle X_{\infty} ; \pi_{\alpha}\right\rangle_{a} \in_{A}$ is a direct limit, then $\bigcup\left\{\pi_{\alpha}\left[X_{\alpha}\right] \mid \alpha \in A\right\}$ generates $X_{\infty}$.

The proof is omitted since it is like the proof of a corresponding result in [2]. We observe that if the operations are finitary for the algebras in the above lemma, then actually $\bigcup\left\{\pi_{\alpha}\left[X_{\alpha}\right] \mid \alpha \in A\right\}=X_{\infty}$.

THEOREM 2.2. Let $\mathfrak{U}$ be a class of algebras of type $\kappa$ with finitary operations. Assume $\mathfrak{A}$ is closed under the formation of subalgebras. Let $\langle L ; \Pi\rangle$ be a direct system over $A$ in $\mathfrak{1}$. Suppose every algebra $L_{\alpha}$ satisfies a sentence of the form

$$
\forall x \exists y R(x, y),
$$

where $R(x, y)$ is some conjunction of identities. Then if $\left\langle L_{\infty} ; \pi_{\alpha}\right\rangle_{a} \in_{A}$ is the direct limit of $\langle L ; \Pi\rangle, L_{\infty}$ also satisfies this sentence.

Proof. By Lemma 2.1, $\bigcup\left\{\pi_{\alpha}\left[L_{\alpha}\right] \mid \alpha \in A\right\}=L_{\infty}$. We notice that for any finite set of elements $x_{1}, \cdots, x_{n}$ in $L_{\infty}$, there exist an $\alpha \in A$ and elements $y_{1}, \cdots, y_{n}$ in $L_{\alpha}$ such that $\pi_{\alpha}\left(y_{i}\right)=x_{i}$ for all $i=1, \cdots, n$. Moreover, if $R(x, y)$ for $x$ and $y$ in $L_{\alpha}$, then $R\left(\pi_{\alpha}(x), \pi_{\alpha}(y)\right)$.

Let $L$ and $L^{\prime}$ be lattices containing 0 and 1 . For a lattice homomorphism $h: L \rightarrow L^{\prime}$, assume, that $h(0)=0$ and $h(1)=1$.

CoRollary 2.3. Let $\mathfrak{A}$ be the class of all lattices and let $\mathfrak{B}$ be the class of complemented lattices. Let $\left\langle L_{\infty} ; \pi_{\alpha}\right\rangle_{\alpha} \in_{A}$ be the direct limit in $\mathfrak{A}$ of a direct system $\langle L ; I\rangle$ over $A$ in $\mathfrak{B}$. Then $\left\langle L_{\infty} ; \pi_{\alpha}\right\rangle_{a} \in_{A}$ is the direct limit of $\langle L ; \Pi\rangle$ in $\mathfrak{B}$.

Proof. Let $\alpha, \beta \in A$. Choose $\delta>\alpha, \beta$. Then

$$
\pi_{\alpha}\left(0_{\alpha}\right)=\pi_{\delta} \pi_{\alpha}^{\delta}\left(0_{\alpha}\right)=\pi_{\delta}\left(0_{\delta}\right)=\pi_{\delta} \pi_{\beta}^{\delta}\left(0_{\beta}\right)=\pi_{\beta}\left(0_{\beta}\right) .
$$

It follows that $L_{\infty}$ has a zero element. Similarly $L_{\infty}$ has a unit element. Thus every $L_{\alpha}$ and $L_{\infty}$ belong to the class 5 of lattices which contain 0 and 1. Moreover, $\left\langle L_{\infty} ; \pi_{\alpha}\right\rangle_{a} \in_{A}$ is the direct limit of $\langle L ; \Pi\rangle$ in $\sqrt{5}$. Every $L_{\alpha}$ satisfies the sentence $\forall x \exists y(x \vee y=1$ and $x \wedge y=0$ ). By Theorem 2.2, $L_{\infty}$ satisfies this sentence and thus is in $\mathfrak{B}$. 
CoRollary 2.4. Let $\mathfrak{A}$ be a class of lattices which contains the class $\mathfrak{B}$ of relatively complemented lattices. Assume that $\mathfrak{A}$ is closed under the formation of sublattices. Let $\langle L ; \Pi\rangle$ be a direct system over $A$ in $\mathfrak{B}$. Then if $\left\langle L_{\infty} ; \pi_{\alpha}\right\rangle_{\alpha} \in_{A}$ is the direct limit of $\langle L ; \Pi\rangle$ in $\mathfrak{A},\left\langle L_{\infty} ; \pi_{\alpha}\right\rangle_{a} \in_{A}$ is the direct limit of $\langle L ; \Pi\rangle$ in $\mathfrak{B}$.

Proof. Every $L_{\alpha}$ satisfies the sentence $\forall a \forall b \forall x \exists y(b \wedge x \wedge y=a$ and $a \vee x \vee y=b$ ). Hence by Theorem 2.2, $L_{\infty}$ satisfies this sentence and thus is in $\mathfrak{B}$.

For the remainder of this section, we consider the existence of direct limits in certain classes of Boolean algebras. A Boolean algebra is a complemented distributive lattice. Let $B$ be a Boolean algebra and let $X(B)$ be the set of all prime ideals of $B . \quad X(B)$ can be topologized in such a way that the set $F(B)$ of all both open and closed sets in $X(B)$ forms a base for the topology. Moreover, $F(B)$ is a field of sets which is isomorphic to $B$. If $\pi$ is homomorphism of $B$ into a Boolean algebra $C$, then $\pi^{-1}[M]$ is a prime ideal of $B$, where $M$ is a prime ideal of $C$. Thus $\pi$ induces a continuous mapping $\pi^{*}: X(C) \rightarrow X(B)$ by letting $\pi^{*}(M)=\pi^{-1}[M]$. In fact, if $U \in F(B)$, $\left(\pi^{*}\right)^{-1}[U]=i \pi h^{-1}(U)$ where $i$ and $h$ are the isomorphisms mapping $C$ onto $F(C)$ and $B$ onto $F(B)$ respectively.

It can be shown that $\pi^{*}$ is one-to-one if and only if $\pi$ is onto and $\pi^{*}$ is onto if and only if $\pi$ is one-to-one.

$X(B)$ is called the Stone space of $B$. For a more detailed discussion of this concept, see [6].

THEOREM 2.5. Let $X$ be any abstract algebra with finitary operations. Suppose $Y=\left\{X_{\alpha} \mid \alpha \in A\right\}$ is a family of subalgebras of $X$ which is directed by inclusion and such that $X=\cup_{\alpha_{A}} X_{\alpha}$. Let $\mathfrak{A}$ be a class of algebras with finitary operations which contains $X$ and $X_{\alpha}$ for all $\alpha \in A$. $A$ is directed by $\alpha<\beta$ if and only if $X_{\alpha} \subset X_{\beta}$. For $\alpha<\beta$, let $\pi_{\alpha}$ and $\pi_{\alpha}^{\beta}$ be the inclusion maps of $X_{\alpha}$ into $X$ and $X_{\alpha}$ into $X_{\beta}$ respectively. Then $\langle Y ; \Pi\rangle$ is a direct system over $A$ and $\left\langle X ; \pi_{\alpha}\right\rangle_{\alpha} \in_{A}$ is the direct limit of $\langle Y ; \Pi\rangle$, where $\Pi=\left\{\pi_{\alpha}^{\beta} \mid \alpha<\beta\right\}$.

Proof. Since $\alpha<\beta<\delta$ implies $X_{\alpha} \subset X_{\beta} \subset X_{\delta}$, it follows that $\pi_{\beta}^{\delta} \pi_{\alpha}^{\beta}=\pi_{\alpha}^{\delta}$. Thus, $\langle Y ; \Pi\rangle$ is a direct system over $A$. Obviously $\pi_{\beta} \pi_{\alpha}^{\beta}=\pi_{\alpha}(\alpha<\beta)$. Since

$$
X=\bigcup_{\alpha \in A} X_{\alpha}, X=\bigcup\left\{\pi_{\alpha}\left[X_{\alpha}\right] \mid \alpha \in A\right\} .
$$

If $h_{\alpha}: X_{\alpha} \rightarrow Z$, where $Z \in \mathfrak{A}$, is a family of homomorphisms such that $h_{\beta} \pi_{\alpha}^{\beta}=h_{\alpha}$, then $\left.h_{\beta}\right|_{x_{\alpha}}=h_{\alpha}$ for $\alpha<\beta$. Define $h: X \rightarrow Z$ as follows: for 
$x \in X$, choose $\alpha_{0} \in A$ such that $x \in X_{\alpha_{0}}$; let $h(x)=h_{\alpha_{0}}(x)$. If $x \in X_{\alpha} \cap X_{\beta}$, choose $\delta \in A$ such that $\alpha<\delta$ and $\beta<\delta$. Then $x \in X_{\delta}$ and $h_{\alpha}(x)=$ $h_{\delta}(x)=h_{\beta}(x)$ since $\left.h_{\delta}\right|_{x_{\alpha}}=h_{\alpha}$ and $\left.h_{\delta}\right|_{x_{\beta}}=h_{\beta}$. This implies $h$ is well defined. Let $x_{0}, \cdots, x_{n}$ be a sequence of elements of $X$. Then

$$
\begin{aligned}
h 0_{n}\left\langle x_{0}, \cdots, x_{n}\right\rangle & =h_{\alpha} 0_{n}\left\langle x_{0}, \cdots, x_{n}\right\rangle \\
& =0_{n}\left\langle h_{\alpha}\left(x_{0}\right), \cdots, h_{\alpha}\left(x_{n}\right)\right\rangle=0_{n}\left\langle h\left(x_{0}\right), \cdots, h\left(x_{n}\right)\right\rangle,
\end{aligned}
$$

where $\alpha$ is such that $x_{0}, \cdots, x_{n} \in X_{\alpha}$. Thus $h$ is a homomorphism. If $x \in X_{\alpha}$, then $\pi_{\alpha}(x)=x$ and $h(x)=h_{\alpha}(x)$ implies $h \pi_{\alpha}=h_{\alpha}$. By 2.1, $h$ is unique. Hence $\left\langle X ; \pi_{\alpha}\right\rangle_{\alpha} \in_{A}$ is the direct limit of $\langle Y ; \Pi\rangle$.

Corollary 2.6. Let $B$ be any Boolean algebra. Let $\mathfrak{B}$ be a class of Boolean algebras which contains $B$ and the set $\bar{B}=\left\{\beta_{\alpha} \mid \alpha \in A\right\}$ of all finite subalgebras of $B$. Let $<, \pi_{\alpha}^{\beta}$, and $\pi_{\alpha}$ be defined as in 2.5. Then $A$ is directed by $\langle$ and $\langle\bar{B} ; \Pi\rangle$ is a direct system over $A$. Moreover, $\left\langle B ; \pi_{\alpha}\right\rangle_{\alpha \in A}$ is the direct limit of $\langle\bar{B} ; \Pi\rangle$.

Proof. For $\alpha, \beta \in A$, let $C$ be the subalgebra of $B$ generated by $B_{\alpha} \cup B_{\beta}$. Then $C$ is finite and $B_{\alpha} \subset C, B_{\beta} \subset C$. Hence $\bar{B}$ is directed by inclusion. Since for each $b \in B$, the subalgebra generated by $\{b\}$ is finite, $B=\bigcup_{\alpha \in A} B_{\alpha}$. Hence the result follows from 2.5.

Let $B$ be a Boolean algebra. An element $b \in B$ is an atom if the ideal generated by $-b$ (that is, the set of all $a \in B$ such that $a \wedge b=0$ ) is maximal ideal. $B$ is said to be atomic provided that for each element $a \neq 0(a \in B)$, there is an atom $b<a$. Observe that every finite Boolean algebra is complete and atomic.

Theorem 2.7. Let $B, \bar{B}$ and $\mathfrak{B}$ be as in 2.6. Suppose that the class $\mathfrak{B}$ contains the class $\mathbb{E}$ of all complete, atomic Boolean algebras. Then $\langle\bar{B} ; I\rangle$ does not admit a direct limit in $\sqrt{5}$ if $B$ is not in $\sqrt{5}$.

Proof. Suppose there is a $C \in \sqrt{ }$ and mappings $\bar{\pi}_{\alpha}: B_{\alpha} \rightarrow C$ such that $\left\langle C ; \bar{\pi}_{\alpha}\right\rangle_{\alpha \in A}$ is the direct limit of $\langle\bar{B} ; \Pi\rangle$. Then there is a unique homomorphism $i: B \rightarrow C$ such that $i \pi_{\alpha}=\bar{\pi}_{\alpha}$. Let $B_{0}=\{0,1\}$. Then $X\left(B_{0}\right)$ contains one point, say $x_{0}$. For all $x \in X(B)$, define $h_{\alpha x}^{*}: X\left(B_{0}\right) \rightarrow$ $X\left(B_{\alpha}\right)$ by $h_{\alpha x}^{*}\left(x_{0}\right)=\pi_{\alpha}^{*}(x)$ for each $\alpha \in A$. There exist unique homomorphisms $\bar{h}_{x}: C \rightarrow B_{0}$ and $h_{x}: B \rightarrow B_{0}$ such that $\bar{h}_{x} \bar{\pi}_{\alpha}=h_{\alpha x}$ and $h_{x} \pi_{\alpha}=$ $h_{\alpha x}$. Also $\bar{h}_{x} i \pi_{\alpha}=\bar{h}_{x} \bar{\pi}_{\alpha}=h_{\alpha x}$ implies $\bar{h}_{x} i=h_{x}$ by the uniqueness of $h_{x}$.

(1) $\left\{\pi_{\alpha}^{*} \mid \alpha \in A\right\}$ separates points.

Let $x \neq y$ be in $X(B)$. Considering $x$ and $y$ as prime ideals of $B$, there is a $b \in B$ such that $b$ is in $x$ and $b$ is not in $y$. There is an $\alpha \in A$ such that $\pi_{\alpha}(b)=b$. This implies $b \in \pi_{\alpha}^{-1}[x]$ and $b$ is not in $\pi_{\alpha}^{-1}[y]$. However, $\pi_{\alpha}^{*}(x)=\pi_{\alpha}^{-1}[x]$ and $\pi_{\alpha}^{*}(y)=\pi_{\alpha}^{-1}[y]$. Hence $\pi_{\alpha}^{*}(x) \neq \pi_{\alpha}^{*}(y)$. 
(2) $\bar{\pi}_{\alpha}^{*}$ is onto.

Let $x_{\alpha} \in X\left(B_{\alpha}\right)$. Since $\pi_{\alpha}^{*}$ is onto, there is $x \in X(B)$ such that $\pi_{\alpha}^{*}(x)=x_{\alpha}$. Thus $h_{\alpha x}^{*}\left(x_{0}\right)=x_{\alpha}$. Now $\bar{\pi}_{\alpha}^{*} \bar{h}_{x}^{*}=h_{\alpha x}^{*}$. Hence $\bar{\pi}_{\alpha}^{*}\left(\bar{h}_{x}^{*}\left(x_{0}\right)\right)=h_{\alpha x}^{*}\left(x_{0}\right)=x_{\alpha}$ and $\bar{\pi}_{\alpha}^{*}$ is onto. This implies $\bar{\pi}_{\alpha}$ is one-to-one and hence $i$ is one-to-one.

(3) If $y \in X(C)$, there exists $x \in X(B)$ such that $\bar{h}_{x}^{*}\left(x_{0}\right)=y$. Let $y \in X(C)$. Define $\bar{h}^{*}: X\left(B_{0}\right) \rightarrow X(C)$ by $\bar{h}^{*}\left(x_{0}\right)=y$. Then $\pi_{\alpha}^{*}(y)=$ $\pi_{\alpha}^{*} i(y)=h_{\alpha i^{*}(y)}\left(x_{0}\right)$. Thus $\bar{\pi}_{\alpha}^{*} \bar{h}^{*}=h_{\alpha i^{*}(y)}$. By the uniqueness of $\bar{h}_{i^{*}(y)}$, $\bar{h}^{*}=\bar{h}_{i^{*}(y)}^{*}$.

(4) $i^{*}$ is one-to-one.

Let $x \neq y$ be in $X(C)$. By (3), there exist $x_{1}, x_{2} \in X(B)$ such that $\bar{h}_{x_{1}}^{*}\left(x_{0}\right)=x$ and $\bar{h}_{x_{2}}^{*}\left(x_{0}\right)=y$. Now $x_{1} \neq x_{2}$. If $i^{*}(x)=i^{*}(y), \pi_{\alpha}^{*}\left(x_{1}\right)=\pi_{\alpha}^{*}\left(x_{2}\right)$ for all $\alpha$ since

$$
\pi_{\alpha}^{*}\left(x_{1}\right)=h_{\alpha x_{1}}^{*}\left(x_{0}\right)=\bar{\pi}_{\alpha}^{*} \bar{h}_{x_{1}}^{*}\left(x_{0}\right)=\pi_{\alpha}^{*} i^{*}(x)=\pi_{\alpha}^{*} i^{*}(y)=\bar{\pi}_{\alpha}^{*} \bar{h}_{x_{2}}^{*}\left(x_{0}\right)=\pi_{\alpha}^{*}\left(x_{2}\right) .
$$

By (1) this is impossible. Hence $i^{*}(x) \neq i^{*}(y)$.

(2) and (4) imply that $i$ is an isomorphism and hence $B$ is isomorphic to $C$.

CoRollary 2.8. Let $\mathfrak{B}$ be a class of Boolean algebras such that

(1) $\mathfrak{B}$ contains all finite Boolean algebras;

(2) $\mathfrak{B}$ does not contain all Boolean algebras;

(3) any algebra isomorphic to an algebra of $\mathfrak{B}$ is in $\mathfrak{B}$. Then $\mathfrak{B}$ is not closed under direct limits.

Proof. Let $\langle\bar{B} ; \Pi\rangle$ and $\left\langle B ; \pi_{\alpha}\right\rangle_{\alpha} \in_{A}$ be as in 2.6 with $B$ not in $\mathfrak{B}$. Then, following the proof of 2.7, if there is an algebra $C \in \mathfrak{B}$ and homomorphisms $\bar{\pi}_{\alpha}: B_{\alpha} \rightarrow C$ such that $\left\langle C ; \bar{\pi}_{\alpha}\right\rangle_{\alpha} \in_{A}$ is the direct limit of $\langle\bar{B} ; \Pi\rangle, C$ is isomorphic to $B$. This is contrary to (3) and the assumption that $B$ is not in $\mathfrak{B}$.

THEOREM 2.9. Let $\mathfrak{B}$ be a class of Boolean algebras which contains the two element Boolean algebra, $B_{0}$. Let $\langle\bar{B} ; \Pi\rangle$ be a direct system over $A$. Suppose

(1) there exists a family of homomorphisms $\pi_{\alpha}: B_{\alpha} \rightarrow B$, where $B \in \mathfrak{B}$, such that if $\alpha<\beta, \pi_{\beta} \pi_{\alpha}^{\beta}=\pi_{\alpha}$;

(2) $\bigcup\left\{\pi_{\alpha}\left[B_{\alpha}\right] \mid \alpha \in A\right\}$ generates $B$;

(3) if $g_{\alpha}: B_{\alpha} \rightarrow B_{0}$ is a family of homomorphisms such that if $\alpha<\beta, g_{\beta} \pi_{\alpha}^{\beta}=g_{\alpha}$, then there exists a unique homomorphism $g: B \rightarrow B_{0}$ such that $g \pi_{\alpha}=g_{\alpha}$.

Then $\left\langle B ; \pi_{\alpha}\right\rangle_{\alpha \in_{A}}$ is the direct limit of $\langle\bar{B} ; \Pi\rangle$.

Proof. Suppose $h_{\alpha}: B_{\alpha} \rightarrow C$, where $C \in \mathfrak{B}$, is a family of homomorphisms such that if $\alpha<\beta, h_{\beta} \pi_{\alpha}^{\beta}=h_{\alpha}$. Let $X\left(B_{0}\right)=\left\{x_{0}\right\}$. For 
$c \in X(C)$, let $g_{\alpha c}^{*}: X\left(B_{0}\right) \rightarrow X\left(B_{\alpha}\right)$ be defined by $g_{\alpha c}^{*}\left(x_{0}\right)=h_{\alpha}^{*}(c)$. Then for every $c \in X(C)$ we have a family of homomorphisms $g_{\alpha c}: B_{\alpha} \rightarrow B_{0}$. Since $\pi_{\alpha}^{\beta^{*}} g_{\beta c}^{*}\left(x_{0}\right)=\pi_{\alpha}^{\beta^{*}} h_{\beta}^{*}(c)=h_{\alpha}^{*}(c)=g_{\alpha c}^{*}\left(x_{0}\right)$, it follows that $g_{\beta_{c}} \pi_{\alpha}^{\beta}=g_{\alpha c}$. By (3), there is a unique homomorphism $g_{c}: B \rightarrow B_{0}$ such that $g_{c} \pi_{\alpha}=$ $g_{\alpha c}$. Define $\mu^{*}: X(C) \rightarrow X(B)$ by $\mu^{*}(c)=g_{c}^{*}\left(x_{0}\right)$. Now

$$
\pi_{\alpha}^{*} \mu^{*}(c)=\pi_{\alpha}^{*} g_{c}^{*}\left(x_{0}\right)=g_{\alpha c}^{*}\left(x_{0}\right)=h_{\alpha}^{*}(c) .
$$

Thus $\mu \pi_{\alpha}=h_{\alpha}$. Since $\bigcup\left\{\pi_{\alpha}\left[B_{\alpha}\right] \mid \alpha \in A\right\}$ generates $B, \bigcup\left\{i \pi_{\alpha} i_{\alpha}^{-1}\left[F_{\alpha}\right] \mid \alpha \in A\right\}$ generates $F$ as a Boolean algebra, where $i: B \rightarrow F$ and $i_{\alpha}: B_{\alpha} \rightarrow F_{\alpha}$ are the isomorphisms of $B$ and $B_{\alpha}$ onto the field of all both open and closed subsets of $X(B)$ and $X\left(B_{\alpha}\right)$ respectively. Hence $\left\{i \pi_{\alpha} i_{\alpha}^{-1}\left[F_{\alpha}\right] \mid \alpha \in A\right\}$ is a subbasis for the topology. Let $U_{\alpha} \in F_{\alpha}$. Then $\left(\pi_{\alpha}^{*}\right)^{-1}\left[U_{\alpha}\right]=i \pi_{\alpha} i_{\alpha}^{-1}\left(U_{\alpha}\right)$. Therefore, $\left(\mu^{*}\right)^{-1}\left[i \pi_{\alpha} i_{\alpha}^{-1}\left(U_{\alpha}\right)\right]=\left(\mu^{*}\right)^{-1}\left(\pi_{\alpha}^{*}\right)^{-1}\left[U_{\alpha}\right]=\left(h_{\alpha}^{*}\right)^{-1}\left[U_{\alpha}\right]$ is open in $X(C)$. Thus $\mu^{*}$ is continuous. By $2.1, \mu: B \rightarrow C$ is unique. Hence $\left\langle B ; \pi_{\alpha}\right\rangle_{\alpha \in A}$ is the direct limit of $\langle\bar{B} ; \Pi\rangle$.

CoROLlary 2.10. Let $\mathfrak{B}$ be a class of Boolean algebras which contains the two element Boolean algebra. Let $\langle\bar{B} ; \Pi\rangle$ be a direct system over A. Suppose $\mathfrak{B}$ satisfies (1) and (3) of 2.9. Assume also that $\mathfrak{B}$ is closed under the formation of subalgebras. Then $\left\langle B ; \pi_{\alpha}\right\rangle_{\alpha} \in_{A}$ is the direct limit of $\langle\bar{B} ; \Pi\rangle$ if and only if $\bigcup\left\{\pi_{\alpha}\left[B_{\alpha}\right] \mid \alpha \in A\right\}$ generates $B$.

Proof. Immediate from 2.1 and 2.9.

\section{REFERENCES}

1. G. Birkhoff, Lattice Theory, Amer. Soc. Colloquium Publications, Vol. 25, rev. ed., New York, 1949.

2. D. J. Christensen, and R. S. Pierce, Free products of $\alpha$-distributive Boolean algebras, Math. Scand. 7 (1959), 81-105.

3. S. Eilenberg, and N. Steenrod, Foundations of Algebraic Topology, Princeton University Press, Princeton, N. J., 1952.

4. Franklin Haimo, Some limits of Boolean algebras, Proc. Amer. Math. Soc. 2 (1951), 556-576.

5. G. C., Hewitt, The existence of free unions in classes of abstract algebras, Proc. Amer. Math. Soc. 14 (1963), 417-422.

6. R. Sikorski, Boolean Algebras, Springer.Verlag, Berlin, 1960.

7. _ - Products of abstract algebras, Fund. Math. 39 (1952), 211-228.

Received May 23, 1966. This paper is a part of the author's doctoral dissertation submitted to the University of Washington.

UNIVERSITY OF MONTANA 



\section{PACIFIC JOURNAL OF MATHEMATICS}

\section{EDITORS}

\section{H. SAMELSON}

Stanford University

Stanford, California

J. P. JANS

University of Washington

Seattle, Washington 98105
J. Dugundji

University of Southern California Los Angeles, California 90007

RICHARD ARENS

University of California

Los Angeles, California 90024

\section{ASSOCIATE EDITORS}
E. F. BECKENBACH
B. H. NeumanN
F. WOLF
K. YosidA

\section{SUPPORTING INSTITUTIONS}

UNIVERSITY OF BRITISH COLUMBIA

CALIFORNIA INSTITUTE OF TECHNOLOGY

UNIVERSITY OF CALIFORNIA

MONTANA STATE UNIVERSITY

UNIVERSITY OF NEVADA

NEW MEXICO STATE UNIVERSITY

OREGON STATE UNIVERSITY

UNIVERSITY OF OREGON

OSAKA UNIVERSITY

UNIVERSITY OF SOUTHERN CALIFORNIA
STANFORD UNIVERSITY

UNIVERSITY OF TOKYO

UNIVERSITY OF UTAH

WASHINGTON STATE UNIVERSITY

UNIVERSITY OF WASHINGTON

AMERICAN MATHEMATICAL SOCIETY CHEVRON RESEARCH CORPORATION TRW SYSTEMS

NAVAL ORDNANCE TEST STATION 


\section{Pacific Journal of Mathematics \\ Vol. 22, No. $1 \quad$ January, 1967}

Charles A. Akemann, Some mapping properties of the group algebras of a

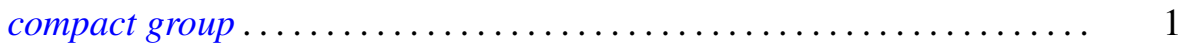

A. V. Boyd, Note on a paper by Uppuluri ..................... 9

Thomas Craig Brown, A semigroup union of disjoint locally finite subsemigroups which is not locally finite .....................

Richard Thomas Bumby and Everett C. Dade, Remark on a problem of

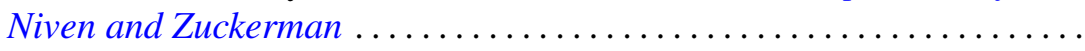

James Calvert, An integral inequality with applications to the Dirichlet problem .........................................

Jack Gary Ceder and Terrance Laverne Pearson, On products of maximally resolvable spaces ....................................

William Guignard Faris, The product formula for semigroups defined by

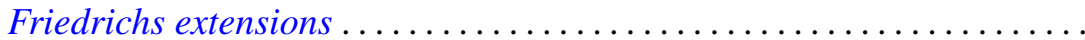

Robert S. Freeman, Closed operators and their adjoints associated with elliptic differential operators ........................ 71

Thomas Lee Hayden, The extension of bilinear functionals ............. 99

Gloria Conyers Hewitt, Limits in certain classes of abstract algebras . . . . . 109

Tilla Weinstein, The dilatation of some standard mappings ........... 117

Mitsuru Nakai, On Evans' kernel ......................... 125

Ernest Levane Roetman, On the biharmonic wave equation ............ 139

Malcolm Jay Sherman, Operators and inner functions ... . .

Walter Laws Smith, On the weak law of large numbers and the generalized elementary renewal theorem

A. J. Ward, On H-equivalence of uniformities: The Isbell-Smith problem 